\title{
TECHNIQUES ET NATURES
}

\section{Pour une approche anthropologique des biomimétismes}

\author{
Perig Pitrou, Lauren Kamili, Fabien Provost
}

Éditions de l'EHESS | «Techniques \& Culture »

$2020 / 1 n^{\circ} 73 \mid$ pages 20 à 33

ISSN 0248-6016

ISBN 9782713228391

Article disponible en ligne à l'adresse :

https://www.cairn.info/revue-techniques-et-culture-2020-1-page-20.htm

Distribution électronique Cairn.info pour Éditions de l'EHESS.

(C) Éditions de l'EHESS. Tous droits réservés pour tous pays.

La reproduction ou représentation de cet article, notamment par photocopie, n'est autorisée que dans les limites des conditions générales d'utilisation du site ou, le cas échéant, des conditions générales de la licence souscrite par votre établissement. Toute autre reproduction ou représentation, en tout ou partie, sous quelque forme et de quelque manière que ce soit, est interdite sauf accord préalable et écrit de l'éditeur, en dehors des cas prévus par la législation en vigueur en France. Il est précisé que son stockage dans une base de données est également interdit. 


\section{Techniques et Natures}

Pour une approche anthropologique des biomimétismes

Perig Pitrou, Lauren Kamili et Fabien Provost

\section{(2) OpenEdition}

Journals

Édition électronique

URL : http://journals.openedition.org/tc/13336

DOI : $10.4000 /$ tc. 13336

ISSN : 1952-420X

Éditeur

Éditions de l'EHESS

\section{Édition imprimée}

Date de publication : 30 juin 2020

Pagination : 20-33

ISBN : 978-2-7132-2839-1

ISSN : 0248-6016

Distribution électronique Cairn

\section{CAIRN INFO}

CHERCHER, REPÉRER, AVANCER.

\section{Référence électronique}

Perig Pitrou, Lauren Kamili et Fabien Provost, «Techniques et Natures », Techniques \& Culture [En ligne], 73 | 2020, mis en ligne le 01 janvier 2023, consulté le 06 juillet 2020. URL : http:// journals.openedition.org/tc/13336 ; DOI : https://doi.org/10.4000/tc.13336 


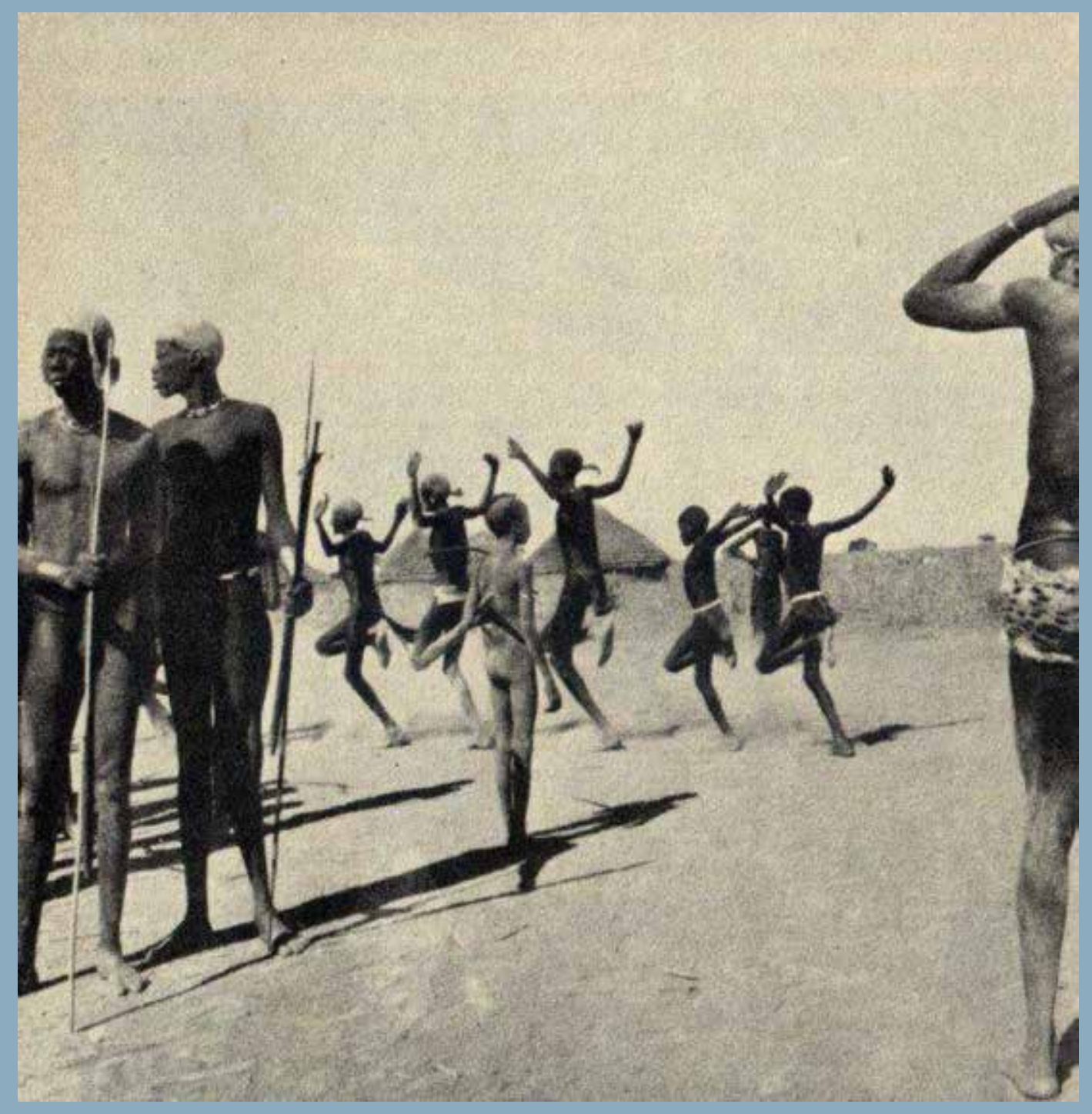

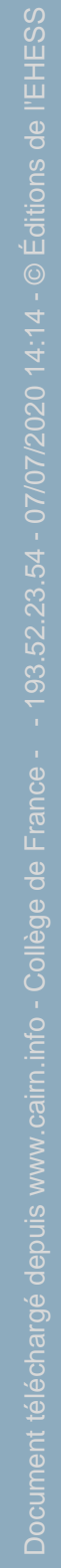




\section{Techniques et Natures}

\section{Pour une approche anthropologique des biomimétismes}

Le biomimétisme désigne un ensemble de démarches et de processus de fabrication qui se proposent d'imiter la nature et les systèmes vivants pour inventer et construire des objets ou des procédés utiles pour les humains. L'imitation pouvant être plus ou moins fidèle ou complète, l'idée de bio-inspiration est souvent mise en avant pour désigner le fait que l'observation de la nature constitue une orientation générale qui stimule des projets techniques. Depuis la parution de Biomimicry (2017 [1997]) de Janine Benyus, le biomimétisme semble s'imposer comme une nouvelle démarche, voire un paradigme révolutionnaire, que les scientifiques et les industriels devraient adopter pour faire des découvertes et développer des projets techniques originaux, moins destructeurs pour l'environnement et capables de favoriser le développement économique. Même si cette démarche se présente comme une solution à la crise écologique, économique et sociale, il importe de ne pas renoncer au temps de la réflexion. La nécessité d'un changement de paradigme technologique pour éviter d'aggraver les catastrophes écologiques et l'extinction des espèces vivantes recommande d'être vigilant et rigoureux au moment d'esquisser les idées directrices pour de nouveaux modèles d'actions individuelles et collectives. La biologiste américaine plaide pour une approche de l'innovation qui respecterait un «cahier des charges du vivant», envisagé sous la forme de neuf «principes du vivant », définis comme « un ensemble de lois, stratégies et principes canoniques » (Benyus 2017 [1997] : 23). Selon ces lois du vivant, la nature fonctionne à l'énergie solaire, valorise l'expertise locale et recycle tout. Pour J. Benyus, il s'agit de considérer la nature non comme une source de matériaux à extraire, mais comme un modèle, un maître dont il faudrait imiter les réalisations. Ces idées concernant la nature sont résumées dans plusieurs formules qui ouvrent son ouvrage: 
1. Avion III (1897) par Clément Ader conçu à partir de l'observation du vol des chauves-souris

Des travaux de Léonard de Vinci aux recherches actuelles d'Airbus sur le faucon et la fabrication de drones dotés d'ailes, il existe une longue filiation entre biomimétisme et aéronautique.

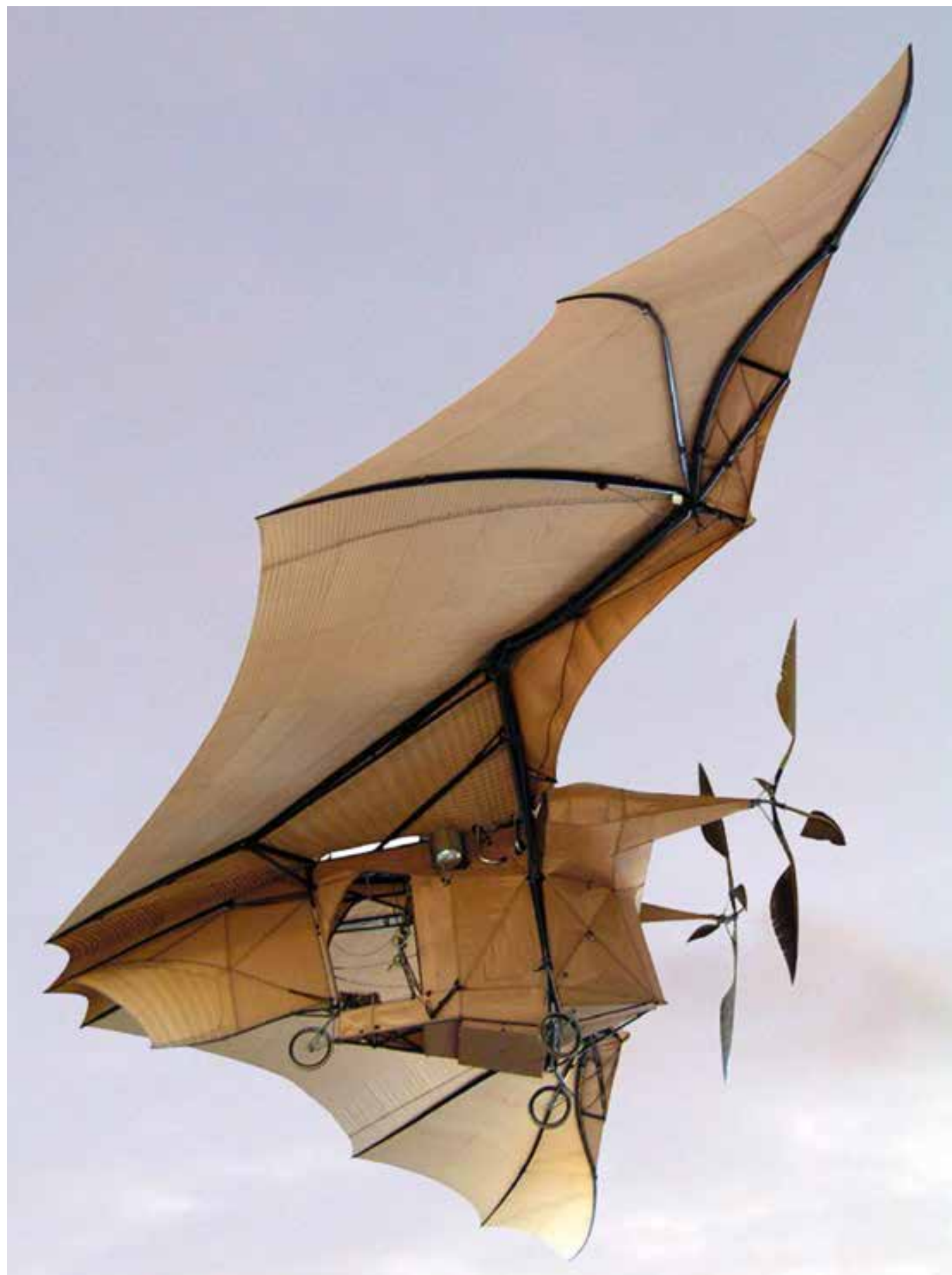


1. La nature comme modèle. Le biomimétisme est une nouvelle science qui étudie les modèles de la nature, puis imite ou s'inspire de ces idées et procédés pour résoudre les problèmes humains [...].

2. La nature comme étalon. Le biomimétisme utilise des critères écologiques pour déterminer si nos innovations sont «bonnes». Au bout de 3,8 milliards d'années d'évolution, la nature a appris à reconnaître ce qui marche [...].

3. La nature comme maître. Le biomimétisme est une nouvelle façon de considérer et d'apprécier la nature. Il ouvre une ère fondée non pas sur ce que nous pouvons extraire du monde naturel, mais sur ce que nous pouvons en apprendre. (Benyus 2017 [1997] : 4)

Ces propositions font du biomimétisme un domaine privilégié pour réfléchir aux relations entre nature et techniques dans une perspective anthropologique, par-delà les oppositions philosophiques entre les projets de maîtrise de la nature par les techniques et leurs critiques (Descartes 1637, Dagognet 1988, Ellul 1954, Heidegger 1993). André Leroi-Gourhan qui décrit l'émergence des techniques dans le monde naturel (1970), les travaux des Science \& Technology Studies (Latour 2001 [1984]), de l'anthropologie de la nature (Descola 1986) ou d'inspiration phénoménologique (Ingold 2000) démontrent que l'étude des processus techniques conduit à dépasser la dichotomie nature/culture, en particulier quand on envisage des pratiques productives (agriculture, élevage, médecine, fermentation, etc.) sous l'angle de leur relation avec les processus vitaux (Coupaye 2013, Pitrou, Coupaye $\&$ Provost 2016). Dans cette perspective, le biomimétisme renvoie à des activités techniques et des conceptions de la nature plus complexes que celles identifiées par Benyus.

Biomimicry invite à poser un regard émerveillé et admiratif sur la perfection de la nature qui «fait» si bien les choses. Cet engouement pour les «merveilles de la nature» s'inscrit en droite ligne dans la tradition de la curiositas qui préside à la constitution des cabinets de curiosités à partir du Xvie siècle. Ces derniers témoignent du «souci d'observer la nature pour mieux la connaître » pour leurs propriétaires qui manifestent leur «admiration pour l'auteur de pareilles merveilles» (Rivallain 2001 : 18-20). À cette même époque, Léonard de Vinci, qui possédait lui-même un cabinet de curiosités, rédige son Codex sur le vol des oiseaux (1505) où le vol des oiseaux est observé pour inventer des machines volantes. De Vinci est souvent présenté comme le premier biomiméticien, ancêtre d'une longue lignée d'ingénieurs qui passe par Gustave Eiffel, architecte en 1889 de la Tour Eiffel reproduisant la structure de l'os du fémur, et Clément Ader, pour son Avion III de 1897 inspiré de la roussette (Wanieck et al. 2017).

La référence à l'émerveillement, récurrente dans les discours standards sur le biomimétisme, souligne que les humains sont guidés par des motivations très diverses lorsqu'ils cherchent à imiter la nature. Le jeu, le rite, l'art autant que la science et l'ingénierie adoptent des points de vue très divers sur le monde naturel pour en objectiver des traits saillants. Jouer à marcher comme un animal, imiter la danse d'un oiseau lors d'un rite nuptial ou faire une ceuvre « d'après nature constituent des pratiques tout aussi biomimétiques que les projets phares d'ordinaire mis en avant. Anthropologiquement, au lieu d'étudier «le» biomimétisme, mieux vaut inventorier les biomimétismes à l'œuvre dans différents contextes sociotechniques. Sous cet angle, on 
2. Joueur de flûte automate et canard de Vaucanson

Ces artefacts illustrent les convictions paradoxales au xvı! siècle: «d'une part on pouvait comprendre la vie et l'intelligence en les reproduisant, et d'autre part que la vie et l'intelligence étaient pré cisément définies par l'impossibilité de les reproduire» (Riskin 2003: 633)

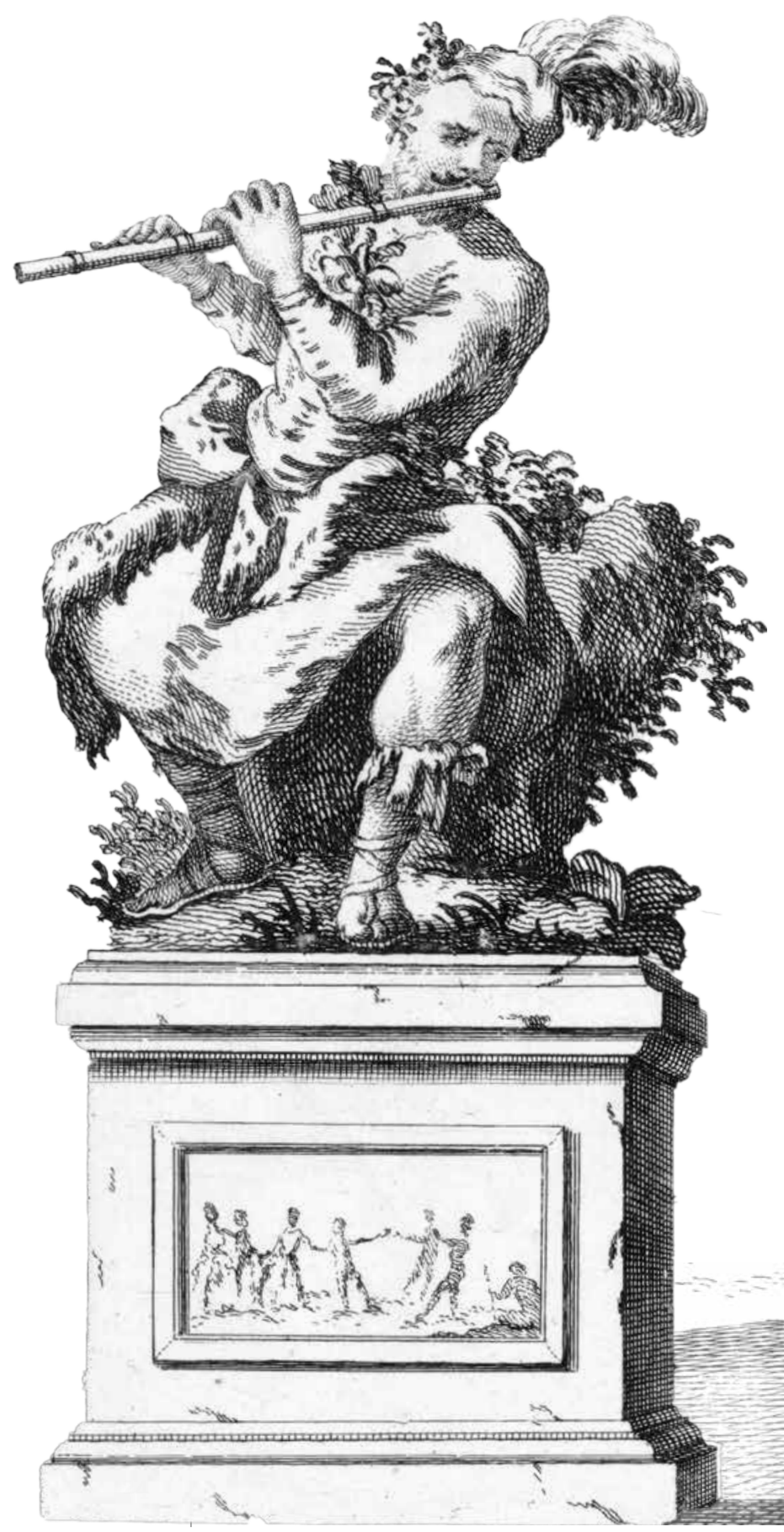

peut appréhender comment l'imitation unit les êtres en instaurant des relations et des attachements très variés entre des humains et des non-humains, sans la réduire à la logique représentationnelle de la mimesis qui repose sur la dichotomie entre l'original et la copie.

\section{Réflexion épistémologique sur un phénomène social}

Présenter l'imitation de la nature comme un moyen de rompre avec des pratiques productivistes explique le succès du biomimétisme. Outre l'abondante bibliographie suscitée par cette thématique, l'approche biomimétique s'institutionnalise dans différents domaines de l'ingénierie et de la recherche scientifique ${ }^{1}$, mais aussi dans l'enseignement et les politiques publiques. Ainsi, l'engouement international pour le biomimétisme est prégnant dans les milieux de l'enseignement supérieur et de la recherche de nombreux pays d'Europe. C'est particulièrement le cas en Allemagne, avec un total de quinze parcours universitaires diplômants ${ }^{2}$. À partir de l'automne 2020, la France comptera aussi deux formations universitaires spécialisées dans le

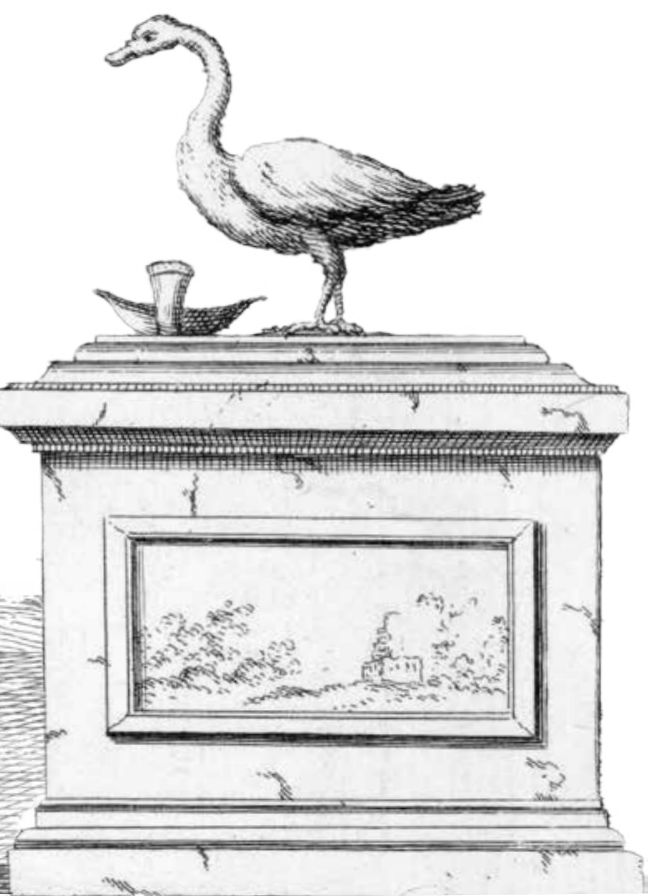


biomimétisme. Cette approche est par ailleurs mentionnée comme levier d'action dans la loi française Biodiversité du 8 août 2016, portée par la ministre Ségolène Royal, et fait aujourd'hui partie intégrante de la stratégie nationale de transition écologique vers un développement durable promue par le ministère de la Transition écologique et solidaire. Enfin, cette démarche est placée au cœur de nombreuses expositions ${ }^{3}$ et expériences de sciences citoyennes ${ }^{4}$. Le biomimétisme est donc un fait social à étudier, non pour faire du biomimétisme mais pour mieux comprendre les relations que les humains établissent avec leur milieu naturel par l'intermédiaire de ce type d'activités techniques.

Dans ce numéro, nous ne souhaitons pas faire l'éloge du biomimétisme: notre intention est de stimuler la réflexion autour de cette notion, approfondissant un travail entrepris lors d'un colloque organisé au Collège de France en 2014 (Pitrou, Dalsuet \& Hurand 2016). Les objectifs poursuivis par le biomimétisme sont si nobles - protéger l'environnement, créer de nouvelles formes de coopération dans les sociétés humaines - qu'il pourrait paraître discordant de sonder la cohérence et la solidité de l'ensemble des propositions et expérimentations associées à cette démarche. Est-on certain que l'imitation de la nature et des systèmes vivants soit une option qui ne comporte aucun inconvénient? A-t-on bien mesuré les conséquences pour l'organisation des sociétés humaines que peut avoir une démarche érigeant la nature comme un modèle à imiter? Comment s'assurer, à l'heure du bilan critique du développement durable et de la croissance verte, que cette option induit des changements effectifs vis-à-vis des procédés techniques caractérisant les sociétés industrielles? Lattrait provoqué par un domaine aussi composite que le biomimétisme -à mi-chemin entre le récit de civilisation, le répertoire de procédés, l'encyclopédie des formes, des matières et des structures, etc. - ne doit pas dispenser de s'interroger sur ses fondements empiriques et sa cohérence théorique. L'adhésion immédiate qu'il suscite devrait même, à bon droit, nous inciter à une certaine prudence épistémologique.

\section{Diversité des techniques biomimétiques}

En tant qu'anthropologues menant des enquêtes ethnographiques sur les conceptions de la vie dans des contextes sociotechniques très divers (sociétés amérindiennes du Mexique, entreprises et laboratoires de science en France, projet Biosphère 2 en Arizona, hôpitaux en France et en Inde), il nous a paru utile de réunir des contributions décrivant un large éventail de pratiques afin de lancer une réflexion collective à partir de données empiriques concrètes. Par contraste avec des approches philosophiques souvent abstraites et textuelles, qui s'attachent à définir ce qu'est ou devrait être le biomimétisme (Mathews 2011, Dicks 2016), la démarche descriptive a l'avantage d'expliciter ce que font les humains lorsqu'ils imitent la nature ou déclarent vouloir le faire. Au lieu de considérer l'imitation comme un mécanisme universel, ou de chercher à tout prix à distinguer biomimétisme et bio-inspiration, et ainsi à en fixer le sens d'un point de vue strictement sémantique, mieux vaut examiner la diversité des techniques impliquées lorsque les humains cherchent à reproduire des systèmes vivants: techniques du corps, techniques cognitives (mesures, fiches, 
mises en cartes, etc.), fabrication et utilisation d'artefacts et de machines, créations d'environnements artificiels. Ouvrir cette boîte noire afin de restituer des chaînes opératoires à l'œuvre pour imiter permet d'éclairer des processus hybrides qui combinent observation, conceptualisation, comptabilisation, figuration, schématisation, fabrication, expérimentation, etc.

Le sous-titre donné au numéro -imitation des êtres vivants et modélisation de la vie-renvoie à deux pôles entre lesquels ranger les opérations techniques mentionnées. Parmi les projets biomimétiques, il est possible de distinguer deux aspects complémentaires, selon que les humains concentrent leur attention sur les organismes pour sélectionner des caractéristiques dans leur morphologie et leur comportement ou qu'ils s'intéressent aux systèmes de relations écologiques qui se tissent autour d'eux. Pour se référer à ce dernier niveau d'organisation, nous suggérons de parler de «modélisation de la vie», une entreprise à ne pas confondre avec «l'imitation des êtres vivants » qui concerne le premier. Dans ce contexte, la vie peut être définie comme un ensemble de causes qui produit les êtres vivants, ces derniers désignant des unités biologiques qui sont en vie.

L'imitation porte sur la reproduction des signes (sons, couleurs, odeurs, etc.), des comportements ou des fonctionnements que les humains peuvent percevoir chez des êtres vivants. Depuis les cérémonies (danses, masques, ornements corporels) jusqu'à la robotique, en passant par les pratiques agricoles, thérapeutiques ou le camouflage employé dans les pratiques cynégétiques, une grande diversité de techniques du corps et d'éléments matériels s'attachent à sélectionner pour les recomposer des caractéristiques morphologiques, physiologiques et fonctionnelles observées dans les organismes. La modélisation de la vie consiste, quant à elle, à reproduire dans des systèmes artificiels les conditions permettant à des êtres vivants d'exister afin de réaliser des expérimentations. Depuis les miniaturisations rituelles des Andes et de la Mésoamérique jusqu'à des dispositifs tels que Biosphère 2, en passant par la construction chez les Achuar de jardins tels des micro-forêts (Descola 1986) ou chez les Maya (Ford \& Nigh 2015), il existe une grande diversité de pratiques de ce type que les enquêtes peuvent documenter.

Notre numéro réunit des études de cas réalisées dans des contextes sociotechniques variés, permettant de mettre en évidence des «théories de la vie» (Pitrou 2019) très diverses selon les organisations sociales. Une série de textes s'appuient sur des matériaux ethnographiques provenant de sociétés traditionnelles d'Océanie (Florence Brunois-Pasina), d'Afrique de l'Est (Jean-Baptiste Eczet), d'Asie centrale (Roberte Hamayon) et de Mésoamérique (Perig Pitrou) pour appréhender diverses manières d'imiter des êtres vivants ou de modéliser la vie. Les analyses de ces auteurs montrent que ces pratiques, souvent réalisées lors de rites, servent des finalités distinctes: techniques agricoles et cynégétiques, construction du corps et de la personne, organisation sociale (classe d'âge, initiation, alliance, etc.), systèmes de collaboration et d'échange avec les non-humains. Les enquêtes dans des laboratoires de science offrent également un éventail de projets s'inspirant des systèmes vivants pour mener des expérimentations: inventaire des potentialités de micro-organismes au Muséum national d'histoire naturelle (Mathilde Gallay-Keller), création d'une ouvre cherchant à reproduire le chant des poissons (Lia Giraud, Térence Meunier, Philippe Marmottant $\&$ Nathalie Henrich Bernardoni), fabrication de proto-cellules en microfluidique (Cyrille Jeancolas), élaboration d'une lampe bioluminescente par une biodesigner (Lauren Kamili), découverte des 
«machines moléculaires» dans un laboratoire de chimie (Sacha Loeve), construction d'un robot imitant les mouvements des fourmis (Stéphane Viollet et al.) ou des homards (Elizabeth Johnson). On verra que des problématiques communes se rencontrent dans toutes ces expérimentations. Les enquêtes menées au Japon sur la fermacculture (Yoann Moreau \& Masumi Oyadomari), ou sur la réhabilitation d'une rivière en France (Marie Lusson) soulignent quant à elles la coexistence de projets écologiques dans des pratiques utilisant des techniques de l'Occident naturaliste.

La confrontation de données recueillies dans des contextes sociotechniques si dissemblables affirme la valeur heuristique de l'approche comparatiste. On peut certes assigner une origine récente à la notion de biomimétisme telle qu'elle se développe en Occident ${ }^{5}$. Mais l'anthropologie adopte une perspective plus large. Loin d'être un phénomène inédit, l'imitation des êtres vivants et la modélisation de la vie se manifestent dans un grand nombre de sociétés, à travers des pratiques parfois très anciennes, et surtout bien plus complexes qu'elles ne semblent l'être au premier regard. Et il y a mille manières d'imiter un être vivant. Même les « rites mimétiques » des Aborigènes, qu'Émile Durkheim présente comme des formes primitives d'organisation religieuse, sont des dispositifs sophistiqués qui objectivent des ordres de faits très distincts (formes, processus, comportement, etc.) en s'appuyant sur un vaste répertoire de procédés techniques (Durkheim 1912). Les travaux de Jessica Riskin (2003) sur les automates de Vaucanson

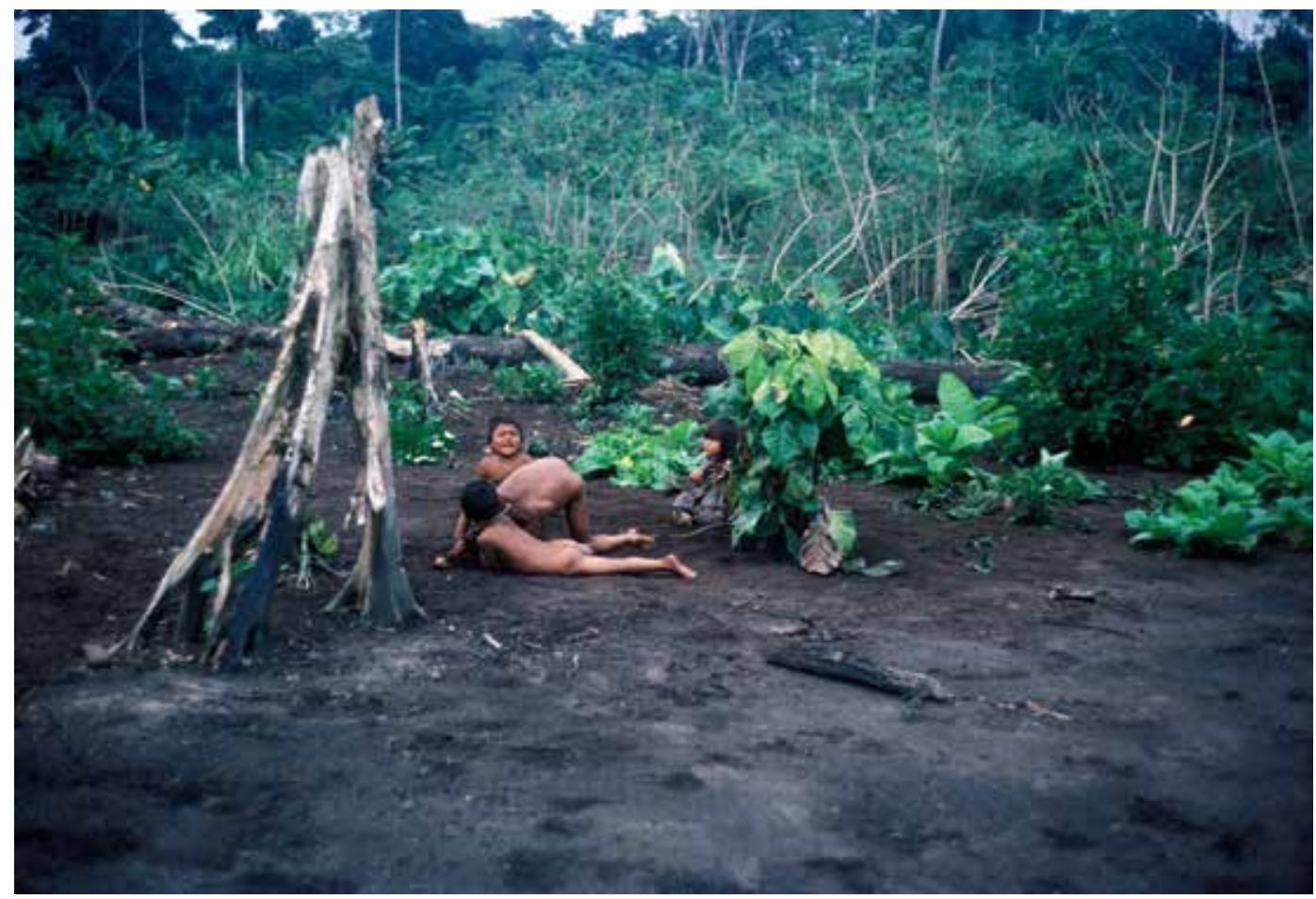

Les similitudes observées entre l'organisation des jardins des Achuar et celle de la forêt de l'Amazonie équatorienne invitent à réfléchir à l'imitation des écosystèmes à partir d'une perspective élargie. Si les jardins peuvent s'envisager comme des microforêts, c'est parce que les forêts sont conçues comme des macrojardins façonnés par des entités non humaines. (Descola 2016) 
soulignent comment les problèmes rencontrés par la science contemporaine, lorsqu'elle cherche à imiter des systèmes vivants, sont déjà au cour de pratiques artisanales. Sans l'anthropologie et l'histoire, on risquerait de ne pas voir la version la plus récente du biomimétisme comme un cas particulier, voire une variante, à considérer à partir d'un cadre plus large.

Deux options sont alors envisageables. On peut réserver le terme de biomimétisme à l'acception qu'il reçoit depuis quelques décennies, en le distinguant des formes d'imitation et de modélisation qui prévalent dans des sociétés traditionnelles -il conviendrait, en toute rigueur, de choisir des termes spécifiques pour désigner chacune de ces formes. Ou alors on décide d'élaborer un concept anthropologique de biomimétisme à partir de la richesse des données empiriques recueillies dans des sociétés très différentes. Nous défendons la deuxième option en affirmant qu'il existe diverses manières de pratiquer et penser le biomimétisme. C'est pourquoi nous proposons de parler de biomimétismes au pluriel.

\section{Innovation technique et diversité des relations à la nature}

Ce numéro de TechniquesECulture est une expérimentation épistémologique qui ambitionne de formuler de nouvelles problématiques pour étudier le biomimétisme (voir Provost, Pitrou, Kamili, ce numéro). Un des enjeux est de déterminer les conceptions de la nature qui correspondent aux «choix techniques» (Lemonnier 1993) effectués dans ce type d'entreprise.

Plusieurs pages pourraient être consacrées à décrypter les soubassements et les implications théoriques des propositions de J. Benyus - rappelées plus haut - tant leur valeur axiomatique et axiologique est discutable. Condensons notre argument. Même si cet auteur et ses épigones souhaitent guider les humains vers un nouveau régime d'interaction avec la nature, ils ne font en réalité que renverser un rapport hiérarchique, sans véritablement renouveler les conceptions fondamentales du naturalisme occidental (Descola 2005). À rebours du projet cartésien de mâ̂trise de la nature, cet idéal biomimétique ou bio-inspiré appelle certes à plus d'humilité, mais il se contente de placer la nature dans la position d'un ingénieur ayant fait des expérimentations pendant des millénaires - les humains devant, symétriquement, occuper une position subordonnée. Or, il est douteux que cette inversion des rôles aide à appréhender la complexité des systèmes naturels et la richesse des expériences humaines réalisées pour interagir avec eux.

D'abord, la conception scientifique d'une nature ingénieure est discutable. Les réalisations des processus physico-chimiques et biologiques ont de quoi fasciner, lorsqu'on voit les structures de verre fabriquées par les diatomées ou celles des coquilles d'escargot. Il serait toutefois erroné de penser que la nature, dans sa totalité, fasse plus qu'atteindre des optimums localisés, lesquels entrent d'ailleurs souvent en tension les uns avec les autres. Il est peut-être instructif d'observer les phénomènes naturels, mais affirmer qu'ils se comportent comme des ingénieurs est plus problématique. Cette métaphore risque de légitimer des raisonnements finalistes, voire des représentations théologiques (Chansigaud 2011). Nous sommes attachés à la conception de l'évolution résumée par 


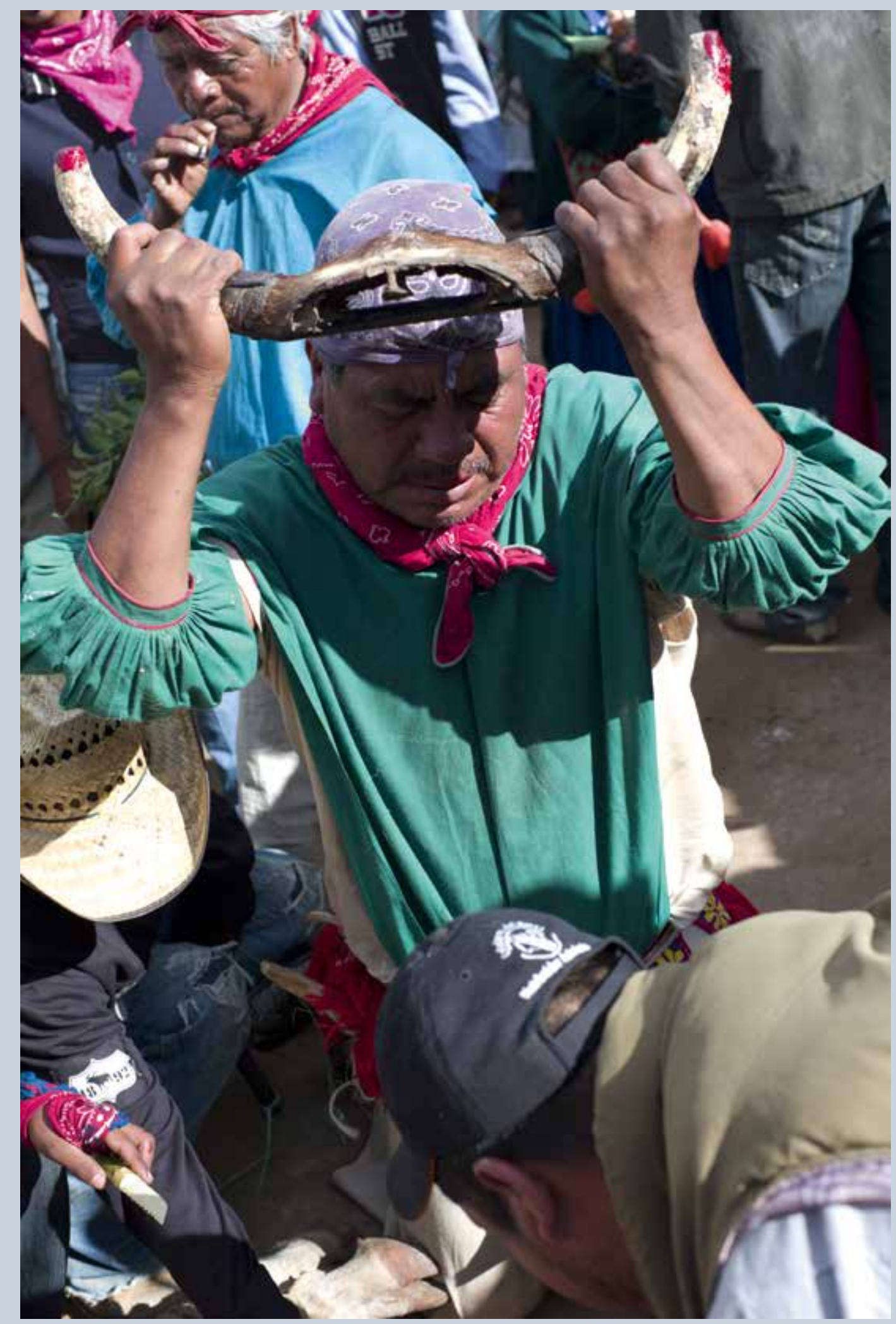


François Jacob dans son article «Evolution and tinkering» (1977). Contre la représentation d'une nature poursuivant un plan, à la manière d'un ingénieur, le biologiste français mobilise, dans le sillage de Claude Lévi-Strauss (1962), la notion de bricolage pour décrire la dimension aléatoire des phénomènes naturels. C'est donc à nos yeux une erreur conceptuelle d'envisager la nature comme une perfection ou comme la manifestation d'un ensemble d'essais et d'erreurs alors que, depuis l'échelle moléculaire jusqu'à l'échelle écosystémique, se manifestent avant tout le hasard et la nécessité. Ce jeu des possibles se rencontre au sein des sociétés humaines dans l'inventivité des techniques mobilisées pour interagir avec les systèmes naturels. Dans Par-delà nature et culture, Philippe Descola (2005) établit comment, à l'instar de l'innovation technique, les formes de collectifs construits par les humains avec les non-humains dépendent des régimes ontologiques au sein desquels ils se développent. Ce n'est pas seulement comparer la nature à un ingénieur qui est discutable: l'idée même d'un domaine uniforme objectivé de façon identique dans toutes les sociétés est erronée. Même si elle inverse la hiérarchie, la conception de la nature de J. Benyus exprime une vision naturaliste occidentale qu'il faut comparer à d'autres ontologies. Qu'est-ce qu'imiter les êtres vivants et modéliser la vie en régime animiste, totémique ou analogiste? La valorisation d'un procédé tel que l'imitation, par essence analogique, dans les procédés naturalistes de la science et de l'ingénierie occidentales, ne traduit-elle pas un bouleversement ontologique en cours ${ }^{6}$ ? Il est crucial d'au moins formuler ces interrogations pour adopter un regard éloigné sur le biomimétisme.

Se concentrer sur ce que font les humains lorsqu'ils imitent la nature au lieu de seulement les y inciter, c'est mettre en regard la dimension évolutive des systèmes naturels et l'inventivité humaine. L'éloge de la nature par le biomimétisme envisage l'évolution uniquement comme une recherche dont les résultats seraient finalisés -comme si d'autres évolutions n'étaient plus envisageables. C'est sans doute la raison pour laquelle les images et les discours les plus standardisés donnent parfois l'impression de réduire la richesse des configurations naturelles à des vues stéréotypées. Il en résulte une récurrence des mêmes récits, des mêmes images: au lieu d'illustrer la fécondité de la nature, cela traduit surtout les schèmes techniciste et fonctionnaliste appliqués pour observer le vivant. L'attention exclusive pour les fonctions occulte le fait que la nature à laquelle les biomiméticiens se réfèrent est elle-même une construction, y compris dans les sciences de la nature: «Nous devons nous souvenir que ce que nous observons n'est pas la nature elle-même, mais la nature exposée à notre méthode de questionnement. » (Werner Heisenberg 1958, T.D.A.)

\section{Ia dynamique biomimétique au-delà des formes fixes et du fonctionnalisme}

La dynamique inhérente à ce dialogue ininterrompu entre techniques et natures oblige à dépasser les vues statiques qui apparient terme à terme des phénomènes naturels et des phénomènes techniques. Le site AskNature ${ }^{7}$ offre un exemple de ce regard qui scrute les êtres naturels à la recherche de solutions pour des problèmes techniques. Plus de 1700 «stratégies biologiques » 
sont ainsi référencées avec presque 200 «idées inspirées » par la nature. Rapportés aux milliards d'êtres vivants qui existent sur Terre, ces chiffres, soit dit en passant, indiquent qu'on est encore loin de générer une encyclopédie biomimétique conçue comme un décalque du grand livre de la nature. La mise en images rend surtout manifeste la dimension analogique du biomimétisme, laquelle fait correspondre des entités provenant du monde naturel avec des objets artificiels.

Or, en dépit du caractère convaincant de ces associations, il convient d'examiner les ressorts d'un projet visant à établir de telles analogies. Le chiasme entre une nature déjà technicienne et une humanité qui devrait naturaliser sa pratique est une position à évaluer. L'élaboration d'un concept anthropologique de biomimétisme ne doit pas seulement restituer les conceptions de la nature et les techniques qui prévalent dans les sociétés humaines: ce cadrage permet aussi de réfléchir aux objectifs non utilitaires et poursuivis dans l'imitation des êtres vivants. Dans un entretien réalisé pour ce numéro, Roberte Hamayon rappelle que la compréhension des métaphores est essentielle pour saisir la complexité des relations que les humains tissent avec leur environnement. Le rite comme le jeu -et, en somme, toutes les constructions techniques et sociales mobilisant la pensée symbolique - ne doivent pas faire oublier que le domaine des techniques est porteur d'univers de significations bien plus vastes et bien plus riches que la recherche de fonction, l'identification de problèmes et la recherche de solutions.

Pour s'ouvrir à une telle richesse, on ne doit pas se satisfaire d'aborder l'imitation du monde selon la logique de la correspondance: les liens s'établissant par l'intermédiaire de l'imitation doivent être mis en évidence. C'est pourquoi le biomimétisme gagne à être abordé sous un angle pragmatique et interactionnel. Prenons l'exemple d'une communication interspécifique, lors de laquelle un humain va adapter ses mouvements et sa gestuelle avec un animal. L'imitation ne repose nullement sur la copie, dans le registre humain, de ce que font les animaux; elle constitue un terrain commun à partir duquel des attachements et une communauté peuvent s'instaurer. Les analyses des rituels proposés par différents auteurs montrent tout le parti que les sociétés humaines savent tirer du potentiel que recèle l'imitation. Qu'elle permette d'adopter le point de vue et la subjectivité de l'autre ou vise à coordonner les interactions entre des êtres qui demeurent séparés, l'imitation apparaît finalement comme un mode d'action polyvalent pour construire une multiplicité de relations entre humains, mais aussi avec les non-humains.

Parler de biomimétismes, au pluriel, ne consiste pas uniquement à défendre l'idée d'une diversité culturelle, cela permet de souligner qu'il existe plusieurs manières, y compris au sein des mêmes sociétés, d'observer et d'imiter les phénomènes et, surtout, que ces entreprises peuvent recevoir des significations très variées. Ainsi, même s'ils sont moins organisés et structurés que le biomimétisme mis en valeur dans les discours actuels, réservons une place, dans l'analyse, à toute une série de biomimétismes - ludiques, rituels, artistiques, empathiques, etc. - auxquels on ne songe pas de prime abord. Notre espoir est ici de forger un concept de biomimétisme plus solide en réfléchissant aux types de collectifs que les humains établissent quand ils imitent leur environnement naturel et les êtres vivants qui les habitent. 


\section{Notes}

1. Pour une présentation de l'état de l'art: Molina \& Raskin 2018, Chayaamor-Heil, Guéna \& Hannachi-Belkadi 2018, Wanieck, Fayemi, Zollfrank et al. 2017, Pawlyn 2011. Pour une présentation grand public, voir Chapelle \& Decoust 2015. Sur l'ingénierie, voir Roth 1983, Coineau \& Kresling 1987, Vogel 1988, Vincent et al. 2006. Sur la chimie verte, voir Anastas \& Warner 1998.

2. Selon le recensement de 2017 du Centre européen d'excellence en biomimétisme de Senlis (CEEBioS).

3. Outre le grand rassemblement annuel Biomim'Expo désormais organisé à la Cité des sciences et de l'industrie, évoquons les expositions «Biomimétisme. Quand la nature inspire l'innovation » au Muséum d'histoire naturelle de Nîmes (2019), «S'inspirer du vivant : le biomimétisme de Léonard de Vinci à nos jours » au musée de Sologne à Romorantin-Lan-

\section{Iconographie}

Image d'ouverture. Dans Divinity and Experience: The Religion of the Dinka, Godfrey Linhardt publie une photo de jeunes garçons imitant des bovins dans une danse. La courbure des bras est présentée comme une attitude corporelle gracieuse et que les hommes adoptent pour leur propre plaisir lorsqu'ils sont seuls avec leur troupeau. (2003 [1961] NY: Oxford University Press).

\section{Références}

Anastas, P. T. \& J. C. Warner 1998 Green Chemistry: Theory and Practice. Oxford: Oxford University Press.

Benyus, J. M. 2017 [1997] Biomimétisme. Quand la nature inspire des innovations durables. Paris: Rue de l'Échiquier.

Chansigaud, V. 2011 «Analyse. Biomimétisme de Janine Benyus», Pour la science 406: 96.

Chapelle G. \& M. Decoust 2015 Le vivant comme modèle. Paris: Albin Michel.

Chayaamor-Heil, N., Guéna, F. \& N. Hannachi-Belkadi 2018 «Biomimétisme en architecture. État, méthodes et outils», Les Cahiers de la recherche architecturale urbaine et paysagère 1 «Innover?». doi : 10.4000/ craup.309.

Coineau, Y. \& B. Kresling 1987 Les inventions de la nature et la bionique. Paris: Hachette. thenay (2019), «La fabrique du vivant» au centre George Pompidou (2019) ou encore «En vie: Aux frontières du design » à l'Espace fondation EDF (2013). En avril 2020, une exposition sur le thème de l'imitation du vivant devait être présentée à la Cité des sciences et de l'industrie.

4. Par exemple le projet Biotope mené par Helena Amalric à Terre Vivante (Isère) ou les réalisations du Low-tech Lab, une association installée à Concarneau (Finistère).

5. La première occurrence du terme «biomimétisme » (biomimetic) est à trouver chez le biophysicien Otto Schmitt (1969).

6. Nous remercions Pierre-Olivier Dittmar pour cette suggestion.

7. https://asknature.org

1. Musée des Arts et Métiers, Paris. CC by SA.

2. BnF, estampe v. 1749-1750, collection Michel Hennin, domaine public.

3. (C) Philippe Descola.

4. Tuxpan de Bolaños, 2012 @ Ivan Alechine.

Coupaye, L. 2013 Growing Artefacts, Displaying Relationships: Yams, Art and Technology Amongst the Nyamikum Abelam of Papua New Guinea. New-York / Oxford: Berghahn.

Dagognet, F. 1988 La maîtrise du vivant. Paris: Hachette.

Descartes, R. 1992 [1637] Discours de la méthode. Paris: Hatier.

Descola, P. 1986 La nature domestique. Symbolisme et praxis dans l'écologie des Achuar. Paris: Éditions de la Maison des sciences de l'homme.

Descola, P. 2005 Par-delà nature et culture. Paris: Gallimard.

Descola, P. 2016 " "Landscape as Transfiguration". Edward Westermarck Memorial Lecture, October $2015 »$, Suomen Antropologi : Journal of the Finnish Anthropological Society 41 (1) : 3-14. [En ligne] : journal. fi/suomenantropologi/article/view/59038. 
Dicks, H. 2016 "The philosophy of biomimicry», Philosophy \& Technology 29 (3) : 223-243.

Durkheim, É. 1912 Les formes élémentaires de la vie religieuse: le système totémique en Australie. Paris: Félix Alcan.

Ellul, J. 1954 La technique ou l'enjeu du siècle. Paris: A. Colin.

Ford, A. \& R. Nigh 2015 The Maya Forest Garden. Eight Millennia of Sustainable Cultivation of the Tropical Woodlands. Londres / New York: Routledge.

Heidegger, M. 1993 Essais et Conférences. Paris: Gallimard.

Heisenberg, W. 1958 Physics and Philosophy. The Revolution in Modern Science. New York: Harper.

Ingold, T. 2000 The Perception of the Environment: Essays on Livelihood, Dwelling and Skill. Londres: Routledge.

Jacob, F. 1977 «Evolution and tinkering», Science 196 (4295): 1161-1166.

Kamili, L. 2019 «Biomimétisme et bio-inspiration : nouvelles techniques, nouvelles éthiques?», Techniques\&Culture, Varia, Meyer, M. \& P. Pitrou dir. «Anthropologie de la vie et des nouvelles technologies». [En ligne] : journals.openedition.org/tc/9299.

Latour, B. 2001 [1984] Pasteur: guerre et paix des microbes suivi de Irréductions. Paris: La Découverte.

Lemonnier, P. 1993 Technological Choices: Transformation in Material Cultures since the Neolithic. Londres / New York: Routledge.

Leroi-Gourhan, A. 1970 Le Geste et la Parole. Paris : Albin Michel.

Lévi-Strauss, C. 1962 La Pensée sauvage. Paris: Plon.

Mathews, F. 2011 «Towards a deeper philosophy of biomimicry », Organization \& Environment 24 (4) : 364-387.

Molina, A. \& K. Raskin 2018 «Biomimétisme: le vivant, source d'innovation », Le journal de l'École de Paris du management $1: 15-22$.

Pawlyn, M. 2011 Biomimicry in Architecture. Londres: Riba Publishing.

Pitrou, P. 2019 «La voie des techniques et la découverte des théories de la vie chez les Achuar» in G. Cometti,
P. Le Roux, T. Manicone \& N. Martin Au seuil de la forêt. Hommage à Philippe Descola. L'anthropologue de la nature. Paris: Tautem.

Pitrou, P., Coupaye, L. \& F. Provost dir. 2016 Des êtres vivants et des artefacts. L'imbrication des processus techniques et des processus vitaux, Actes du colloque organisé au musée du quai Branly les 9 et 10 avril 2014. [En ligne] : actesbranly.revues.org.

Pitrou, P., Dalsuet, A. \& B. Hurand 2016 « Modélisation, construction et imitation des processus vitaux. Approche pluridisciplinaire du biomimétisme», Natures Sciences Sociétés 23 (4): 380-388.

Riskin, J. 2003 «The defecating duck, or, the ambiguous origins of artificial life», Critical Inquiry 29 (4): 599-633.

Rivallain, J. 2001 «Cabinets de curiosité, aux origines des musées», Outre-mers 88 (332-333) «Collectes et collections ethnologiques : une histoire d'hommes et d'institutions » : 17-35. doi: 10.3406/outre.2001.3878.

Roth, R. R. 1983 «The foundation of bionics», Perspectives in Biology and Medicine 26 (2): 229-242.

Schmitt, O. H. 1969 «Some interesting and useful biomimetic transforms », Proceedings of the Third International Biophysics Congress, Boston, MA, 29 août-3 septembre 1969.

Vincent, J. et al. 2006 «Biomimetics: Its practice and theory», Journal of the Royal Society Interface 3 (9): 471-482.

Vogel, S. 1988 Life's Devices : the Physical World of Animals and Plants. Princeton: Princeton University Press.

Vogel, S. 2000 Cats' Paws and Catapults: Mechanical Worlds of Nature and People. New York: W. W. Norton \& Company.

Wanieck, K., Fayemi, P.-E., Maranzana, N., Zollfrank, C. \& J. Shoshanah 2017 «Biomimetics and its tools», Bioinspired, Biomimetic and Nanobiomaterials 6: 1-52. doi: 10.1680/jbibn.16.00010.

\section{Remerciements}

Notre projet a obtenu le soutien financier du CNRS à travers les programmes de la Mission pour les initiatives transverses et interdisciplinaires (MITI).

\section{Pour citer l'article}

Pitrou, P., Kamili, L. \& F. Provost 2020 «Techniques et Natures. Pour une approche anthropologique des biomimétismes», TechniquesE Culture 73 «Biomimétismes. Imitation des êtres vivants et modélisation de la vie», p. 20-33. 